\title{
Blebbishield emergency program: an apoptotic route to cellular transformation
}

\author{
GG Jinesh ${ }^{\star, 1}$ and AM Kamatt ${ }^{*, 1}$
}

Cell Death and Differentiation (2016) 23, 757-758; doi:10.1038/cdd.2016.26; published online 11 March 2016

Apoptosis is an orderly dismantling process of cells by forming apoptotic bodies, which culminates in secondary necrosis in the absence of phagocytes. ${ }^{1}$ We have found that cancer stem cells undergoing apoptosis can avoid cell death by evoking the 'blebbishield emergency program' to enter cellular transformation. $^{2,3}$ In the blebbishield emergency program, the first visual sign that indicates the course of apoptosis is being reversed is the construction of blebbishields from apoptotic bodies. ${ }^{2}$ Blebbishield construction requires endocytosis and endocytosis-driven serpentine filopodia formation that tether the apoptotic bodies together and facilitate fusion (Figure 1a). ${ }^{2,3}$ Inhibition of dynamin-dependent endocytosis blocks the action of serpentine filopodia, inhibits blebbishield formation, and promotes apoptotic body formation. ${ }^{3}$

These mechanical steps require energy and functional mitochondria to generate adenosine triphosphate (ATP). Transformation-competent blebbishields protect their mitochondria, whereas induction of efficient mitochondrial depolarization abolishes transformation from blebbishields. ${ }^{4}$ Interestingly, we found that mitochondrial oligomers of K-Ras, BAD, p27, Bax, and Bak boost glycolysis to meet the energy demand during blebbishield formation (Figure 1b). ${ }^{5}$ Oligomerization of mitochondrial regulators, especially Bax and Bak, has been implicated in mitochondrial outer membrane permeabilization (MOMP). ${ }^{6}$ However, we have shown that oligomerizations of K-Ras, BAD, p27, Bax, and Bak can also occur in non-apoptotic cells, indicating that these oligomerizations might not be dealing with MOMP as such. ${ }^{5}$ Instead, we found that Bax-p18 (a potent MOMP inducer ${ }^{7}$ ) or cleaved Bak correlates with MOMP and cell death (Figure 1c)..$^{5}$

We found that non-apoptotic cancer cells that exhibit constitutive oligomerization produce approximately ten times more lactate (glycolysis metabolite) than the cells that do not exhibit constitutive oligomerization. ${ }^{5}$ We also found that RT4v6 bladder cancer cells do not exhibit constitutive oligomerization under non-apoptotic conditions, but promote oligomerization during the apoptotic phase of blebbishield formation to increase glycolysis so that blebbishields can prevent secondary necrosis ${ }^{5}$ (a process onsets due to lack of ATP). Phosphorylation of BAD at Ser-112 is known to promote glycolysis and block apoptosis, ${ }^{8}$ whereas dephosphorylation of Ser-112 is needed to enable BAD's apoptotic action. ${ }^{9}$ Caspase-3-mediated degradation of p70S6K and Pim-1 (two prominent kinases that phosphorylate BAD at Ser-112) is required to block transformation from blebbishields (Figure 1c). ${ }^{4,5}$

Although increase in glycolysis during apoptosis can generate more ATP and prevent secondary necrosis, blebbishield formation is just a damage control process, and if blebbishields are left without appropriate survival signals (exogenous and or autocrine VEGF- $\mathrm{A}^{3}$ ), they ultimately will not survive. Internal ribosome entry site (IRES) translation promotes survival signals by IRES translation of VEGF-A and anti-apoptotic C-IAP2 to ignite the resurrection process and to enter cellular transformation (Figures $1 \mathrm{a}$ and b). ${ }^{4}$ IRES translation can occur in apoptotic cells, and IREStranslational targets such as c-IAP2 and N-Myc are capable of tipping the balance towards resurrection. ${ }^{10}$ We found that c-IAP2 was translated in transformation-competent blebbishields generated by the combination of Smac mimetic (AZ58) and FasL. ${ }^{5}$ Equally crucial is the expression of N-Myc (IRES-translational target) and VEGFR2 (signals to induce $\mathrm{N}-\mathrm{Myc}$ translation) because expression of these two molecules are required for transformation from blebbishields. ${ }^{4}$ Neutralizing antibodies to VEGF-A or VEGFR2 ${ }^{3}$ or degradation of VEGFR2 by Smac mimetic in combination with TNF-a or TRAIL ${ }^{4}$ abolishes transformation from blebbishields (Figures 1a and b). Downstream to VEGFR2, p70S6K plays a pivotal role by coordinating serpentine filopodia formation as well as IRES translation to drive the blebbishield emergency program. $^{3}$

Importantly, we implicate transformation from blebbishields as an essential intermediate step of K-Ras-mediated transformation $^{5}$ because K-Ras mediates the metabolic switch to glycolysis ${ }^{11}$ through Pim-1, p27 and N-Myc (Figure 1d). ${ }^{5}$

Cells transformed from blebbishields after apoptosis are capable of tumorigenesis and exhibit elevated drug resistance. ${ }^{1}$ This raises the possibility that cancers that relapse after therapy could utilize the blebbishield emergency program to survive the insult of front-line therapeutics. ${ }^{12}$

\footnotetext{
${ }^{1}$ Department of Urology, Unit 1373, The University of Texas MD Anderson Cancer Center, Houston, TX, USA

*Corresponding author: GG Jinesh or AM Kamat, Department of Urology, Unit 1373, The University of Texas MD Anderson Cancer Center, 1515 Holcombe Boulevard, Houston, TX 77030, USA. Tel: 7135635115 or Tel: 713792 3250; Fax: 713794 4824; E-mail: goodwinjinesh@gmail.com or akamat@mdanderson.org
} 

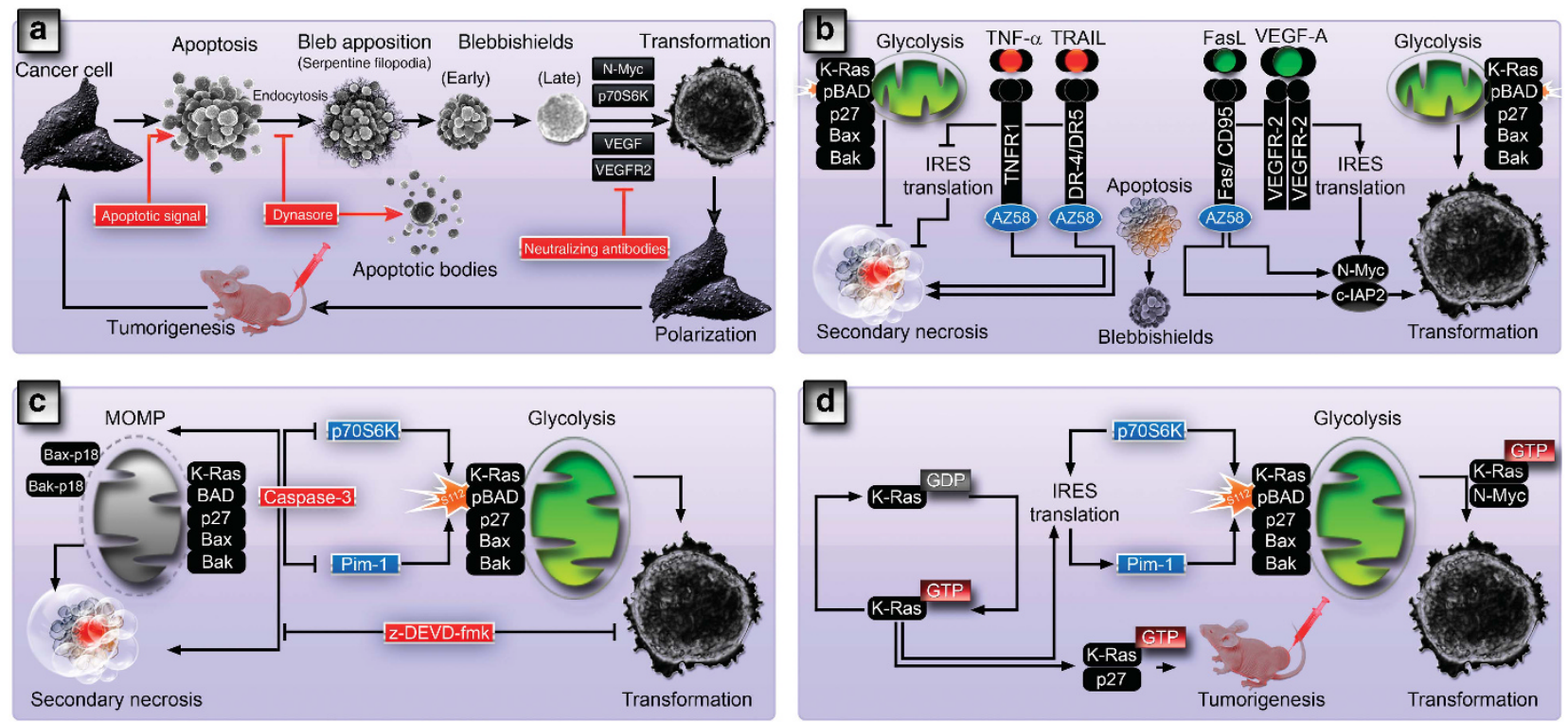

Figure 1 Cell biology and therapeutic targets of the blebbishield emergency program. (a) Cell biological events of the blebbishield emergency program. Endocytosis inhibition blocks blebbishield formation and promote apoptotic body formation, whereas neutralizing antibodies to VEGF or VEGFR2 blocks transformation from blebbishields. (b) Differential regulation of glycolysis and secondary necrosis by Smac mimetic (AZ58) in combination with TNF- $\alpha$, TRAIL, and FasL determines the fate of apoptotic cancer stem cells. Inhibition of IRES translation is critical to block transformation from blebbishields. Note: oligomers depicted could be homo-oligomers and/or hetero-oligomers in nature. (c) Caspase-3 blocks p70S6K and Pim-1 yet required for transformation as z-DEVD-fmk blocks transformation. (d) The blebbishield emergency program is an essential intermediate step in K-Ras-induced transformation as both K-Ras and blebbishields utilize p27, Pim-1, N-Myc and BAD-mediated glycolytic switch for transformation

\section{Conflict of Interest}

The authors declare no conflict of interest.

Acknowledgements. We sincerely thank Ms. Stephanie Deming for editorial assistance.

1. Silva MT. FEBS Lett 2010; $584: 4491$.

2. Jinesh GG et al. Cell Death Differ 2013; 20: 382.
3. Jinesh GG, Kamat AM. Cell Death Discov 2016; 2: 15069.

4. Jinesh GG, Laing NM, Kamat AM. Biochem J 2016; 473: 99.

5. Jinesh GG et al. Cell Death Discov 2016; 2: 16003.

6. Westphal D, Kluck RM, Dewson G. Cell Death Differ 2014; 21: 196.

7. Gao G, Dou QP J Cell Biochem 2000; 80:53.

8. Aho TL et al. FEBS Lett 2004; $571: 43$.

9. Chiang CW et al. Mol Cell Biol 2003; 23: 6350.

10. Jinesh GG, Chunduru S, Kamat AM. J Leukoc Biol 2012; 92: 233.

11. Ying $\mathrm{H}$ et al. Cell 2012; 149: 656.

12. Goodwin Jinesh G. Willis DL, Kamat AM. Curr Stem Cell Res Ther 2014; 9: 89. 